Case Report

\title{
Intracranial Hemorrhage Complicating Herpes Simplex Encephalitis on Antiviral Therapy: A Case Report and Review of the Literature
}

\author{
Ghada ElShimy, ${ }^{1}$ Christina Mariyam Joy, ${ }^{1}$ Fred Berlin, ${ }^{2}$ and Waleed Lashin ${ }^{1}$ \\ ${ }^{1}$ Department of Internal Medicine, St. Joseph's Regional Medical Center, New York Medical College, Paterson, NJ, USA \\ ${ }^{2}$ Department of Diagnostic and Interventional Radiology, St. Joseph's Regional Medical Center, \\ New York Medical College, Paterson, NJ, USA
}

Correspondence should be addressed to Christina Mariyam Joy; christina.mariyam@gmail.com

Received 27 April 2017; Revised 14 July 2017; Accepted 24 July 2017; Published 19 September 2017

Academic Editor: Gernot Walder

Copyright (c) 2017 Ghada ElShimy et al. This is an open access article distributed under the Creative Commons Attribution License, which permits unrestricted use, distribution, and reproduction in any medium, provided the original work is properly cited.

\begin{abstract}
Herpes simplex virus (HSV) encephalitis is the most common cause of nonendemic sporadic encephalitis in the USA. Decreased mortality with early treatment with acyclovir has been documented. Although common complications include cortical petechial hemorrhages, frank intracerebral hematomas are considered very rare. Only few cases have been reported in the literature. We report a case of HSV encephalitis complicated by intracerebral hemorrhage 12 days after initiation of acyclovir therapy.
\end{abstract}

\section{Introduction}

Herpes simplex virus encephalitis is the most common cause of nonendemic sporadic encephalitis in the USA. Mortality can be as high as $70 \%$ when left untreated $[1,2]$. Early initiation of treatment with acyclovir is associated with reduced mortality. However some neurological sequela often persist in these patients. Frank hematoma is extremely rare despite initiation of treatment; only 27 cases have been reported in the literature [2-20].

\section{Case Report}

A 49-year-old male with past medical history of type-1 diabetes mellitus, hepatitis $\mathrm{B}$, chronic hepatitis $\mathrm{C}$, intravenous drug abuse, and traumatic brain injury sustained after fall 2 years prior to presentation (with left hemisphere encephalomalacia) with no history of gastrointestinal bleed (not on aspirin or any anticoagulation treatment at home) presented to the emergency department (ED) with headache, altered mental status, and fever for 2 days' duration.

On arrival, patient was febrile with temperature of $102 \mathrm{~F}$, pulse rate of 112 beats per minute, blood pressure of
$143 / 80 \mathrm{mmHg}$, and respiratory rate of 20 breaths/minute with saturation of $100 \%$ on room air. Physical exam revealed a well-built and nourished gentleman, awake and orientated to person and place with Glasgow coma scale of 15. Fundoscopic exam revealed no papilledema. He was able to move all four extremities but had positive meningeal signs. Laboratory tests showed white blood cell (WBC) count of $9100 / \mathrm{mm}^{3}$ (72\% polymorph nuclear (PMN) leukocytes and $19 \%$ lymphocytes). Patient had a lumbar puncture done and cerebrospinal fluid (CSF) analysis showed WBC count of 45 cells $/ \mathrm{mL}$ (72\% PMN leukocytes and 24\% lymphocytes); protein of $78 \mathrm{mg} / \mathrm{dL}$; and glucose of $201 \mathrm{mg} / \mathrm{dl}$. Noncontrast computed tomography (CT) head showed an old left hemisphere encephalomalacia without any acute intracranial pathology (Figure 1).

Patient was treated empirically for meningoencephalitis (pending cultures) with vancomycin, ceftriaxone, and acyclovir plus first dose of dexamethasone prior to initiation of antibiotics and droplet precaution. Subsequently noncontrast magnetic resonance imaging (MRI) of the brain showed right temporal lobe involvement suggestive of HSV encephalitis (Figure 2). Electroencephalography (EEG) showed slowing in the right frontoparietal area with no 

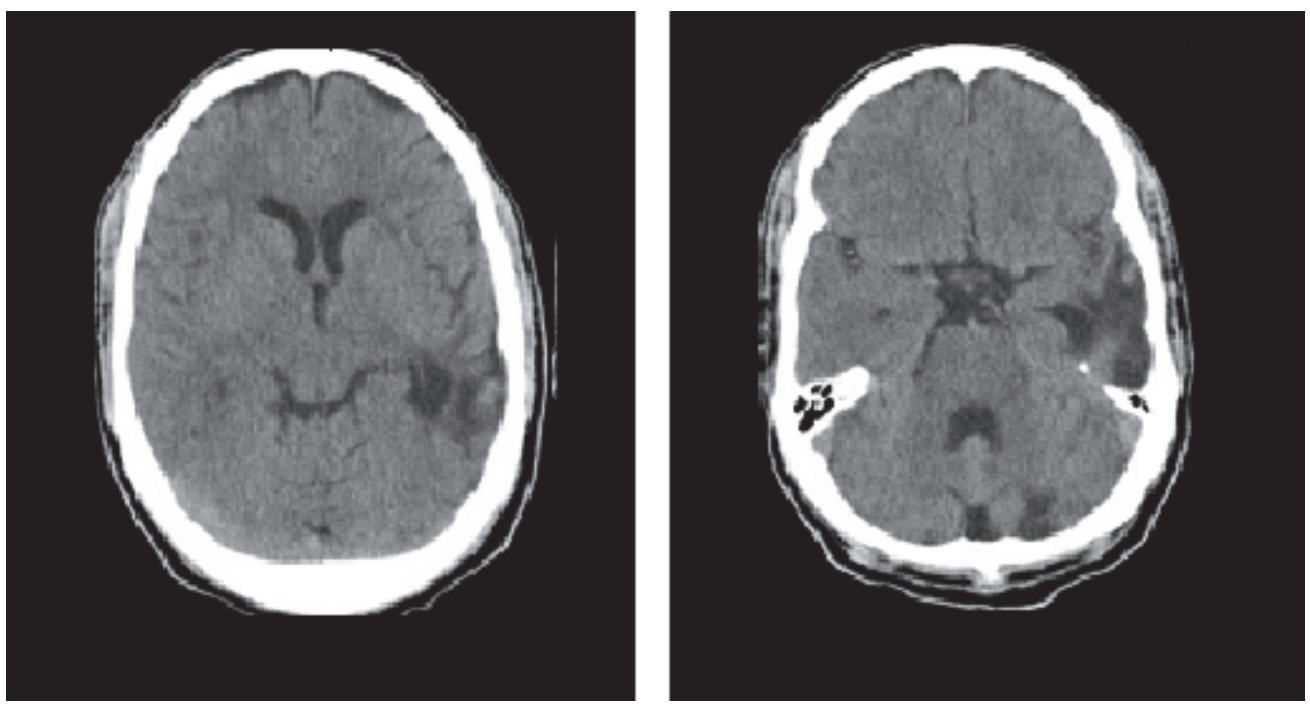

FIGURE 1: (Day 1) noncontrast CT head showing encephalomalacia on the left hemisphere without hemorrhage.

electrographic seizures. Patient's CSF cultures came back negative for both bacteria and HSV deoxyribonucleic acid (DNA) by polymerase chain reaction. But based on the patient's initial clinical presentation, EEG and MRI highly suggestive of HSV encephalitis, antibiotics were discontinued and acyclovir was continued. In the next days, fever subsided and neurological status improved so patient was discharged home on acyclovir treatment.

Twelve days from the initial presentation, patient was brought back to the ED due to acute worsening of mental status. There was no trauma or fall as per family. He was confused, alert but disoriented to person, time, and place, and not following commands. Physical exam did not show any signs of trauma. Laboratory tests showed platelets count 343 $\times 10^{3} / \mathrm{mm}^{3}$, partial thromboplastin time (PTT) 29.1 seconds, prothrombin time (PT) 14.3 seconds, and international normalized ratio (INR) 1.1. Noncontrast CT of the brain demonstrated interval development of acute hemorrhage within medial right temporal lobe, a rare complication of herpes simplex encephalitis. Computed tomography angiography (CTA) done at the same time was negative for any underlying vascular lesion (Figure 3). Neurosurgery team was consulted and patient was treated conservatively. Subsequent CT scans done showed gradual improvement of the hematoma. Patient was continued on IV acyclovir and was discharged. Patient was followed up over the next 2 years and he returned to his baseline neurological status.

\section{Discussion}

Herpes simplex virus encephalitis usually has 2 peaks in incidence (at ages of 20s and 50s). There is no seasonal variation. Risk is not increased in immunocompromised patients; however it is more documented in HIV positive individuals. Our patient had diabetes mellitus, hepatitis B, and hepatitis C but not HIV. HSV type I occurs in vast majority of patients; however few cases can occur with HSV-2. The disease is fatal with very high mortality rate in untreated individual (70\% mortality) with no regain of full neurological function except in $2.5 \%$ of these patients $[1,21,22]$.

The decision to initiate antiretroviral therapy in a patient with suspected viral encephalitis is often clinical. Although serological testing is of prognostic value for identification of specific pathogens, it should not delay the treatment. Detection of antibodies of HSV virus in the CSF may not provide adequate yield especially if the sample is collected early during the convalescent stage of the disease as what occurred in our patient. Hence subsequent testing is helpful [23, 24]. Neurodiagnostic evaluation can provide support for the diagnosis by the demonstration of temporal lobe edema or hemorrhage by magnetic resonance image scan as well as spike and slow-wave activity on electroencephalogram [25]. In our patient, initial HSV PCR was negative; however the clinical scenario, CSF results, MRI, and EEG, all of them, supported the diagnosis of HSV encephalitis especially in the setting of clinical and neurological improvement with acyclovir treatment and resolution of fever in the next days.

In literature, it was documented that MRI is more significant in early detection of HSV encephalitis than CT scan with better sensitivity [26]. Around $90 \%$ of MRI done within the first 48 hours of admission are abnormal in HSV encephalitis. Early finding on MRI usually occurs in the cingulate gyrus and medical temporal lobe. They are characterized by gyral edema on $\mathrm{T} 1$ weighted images and high signal intensity on T2-weighted and T2 FLAIR (87.7\% specificity) [27-29]. Our patient had right medial temporal lobe lesion characteristic of HSV encephalitis.

Other invasive diagnostic tests include brain biopsy which is considered the most sensitive and specific diagnostic tool. However, it is reserved for patients who does not respond to acyclovir therapy and if the etiology cannot be confirmed by CT or MRI that is why it was not required in 


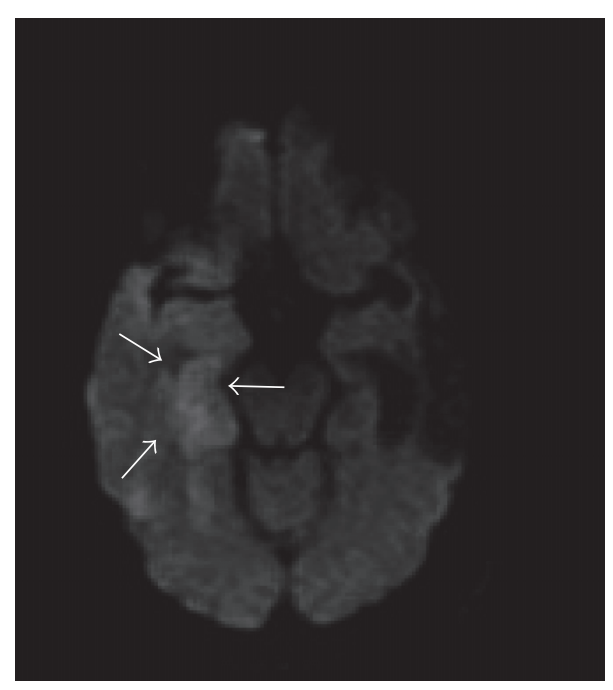

(a)

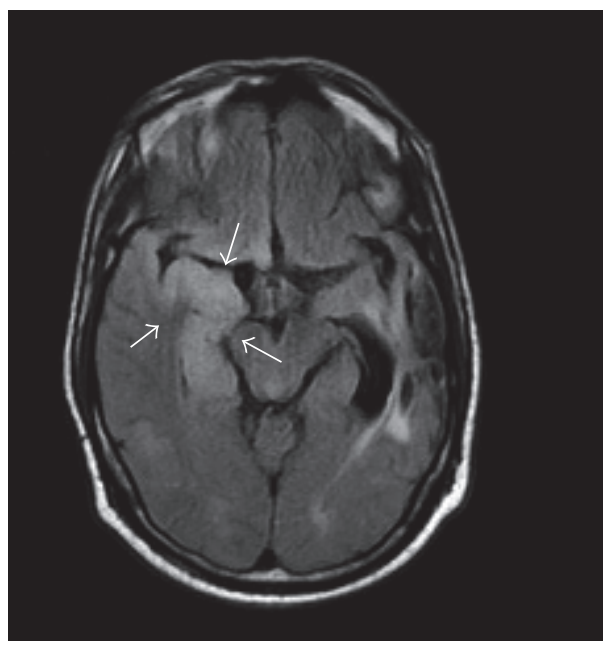

(c)

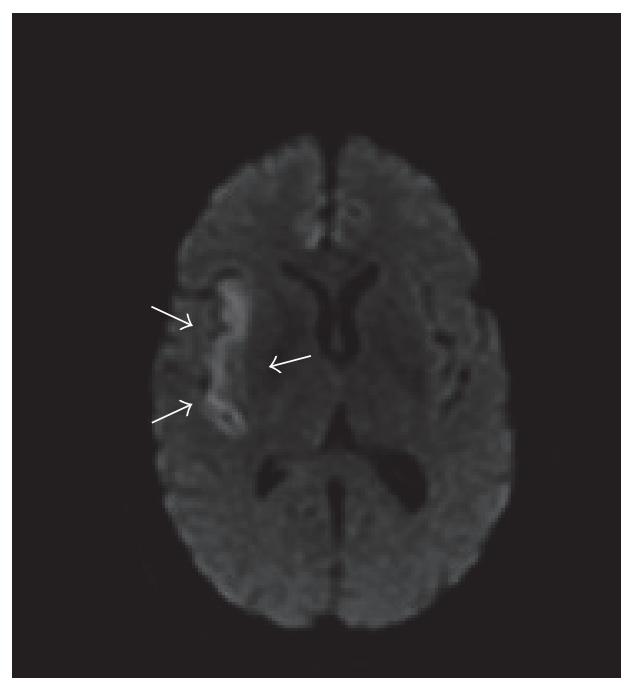

(b)

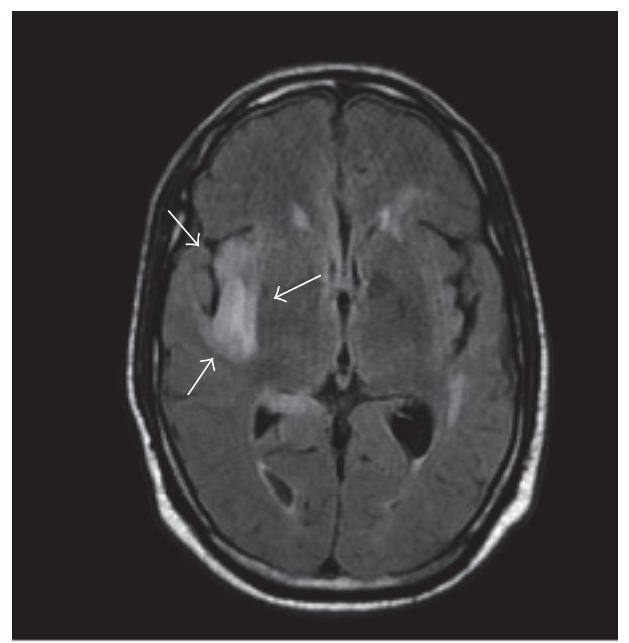

(d)

FIGURE 2: (Day 2) ((a) and (b)) noncontrast MRI of the brain: Diffusion Weighted Imaging (DWI) demonstrates increased signal in the right temporal lobe (a) and right insula (b), consistent with cytotoxic edema. Restricted diffusion is less intense as compared to infarction. These imaging findings in combination with clinical scenario are highly suggestive of herpes simplex encephalitis. ((c) and (d)) Noncontrast MRI of the brain: FLAIR sequence demonstrates asymmetrical involvement of the right medial temporal lobe (c) and the insular cortex (d). Unlike in middle cerebral territory infarct, basal ganglia is spared. Arrows in (a) refer to increased signal in right temporal lobe, in (b) increased signal in right insula, in (c) FLAIR sequence demonstrating involvement of right medial temporal lobe, and in (d) FLAIR sequence demonstrating involvement of right insular cortex.

our patient. There are no specific EEG patterns that are pathognomonic for HSV encephalitis. However some features are helpful in the diagnosis as focal or lateralized EEG abnormalities [30]. Our patient had slowing in right frontoparietal region in the presence of encephalitis which also supported the diagnosis.

Intracranial hemorrhage is a rare complication of herpes encephalitis despite early initiation of antiviral therapy. Necrotizing infiltrate with scattered foci of small hemorrhage in the brain is usually seen; however, frank hematoma is rare and usually occurs during the second week after initial presentation. Intracranial pressure continues to rise and peaks at 11-12 days from onset of illness possibly explaining why our patient developed frank hematoma on day 12 . However, there are other reported cases when bleeding occurred earlier [31]. Our patient had normal platelet count with normal coagulation profile, absent aneurysm, or any vascular abnormalities on CTA and he was not on aspirin or anticoagulation therapy excluding other causes of intracranial hemorrhage. Interestingly the intracerebral hemorrhage was just localized to the area of prior HSV presented 12 days earlier.

The exact mechanism for hemorrhage is unclear. The hemorrhage was proposed to be likely due to small vessel vasculitis resulting in endothelial damage with secondary bleeding. This was explained based on the finding of fibrinoid necrosis in the pathological samples of evacuated hematoma 


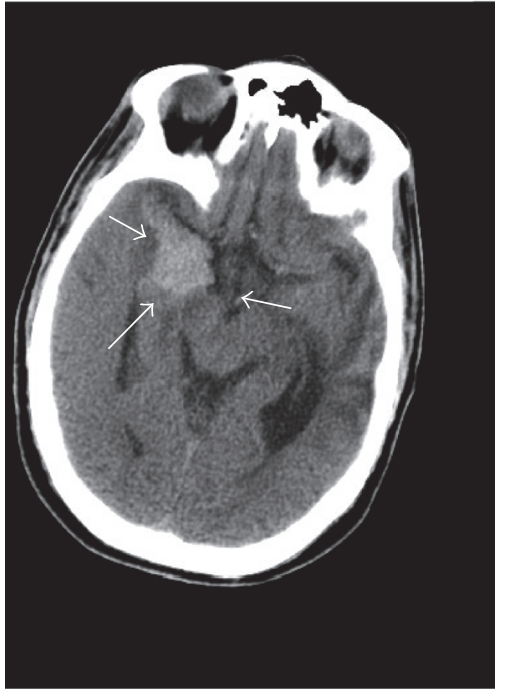

(a)

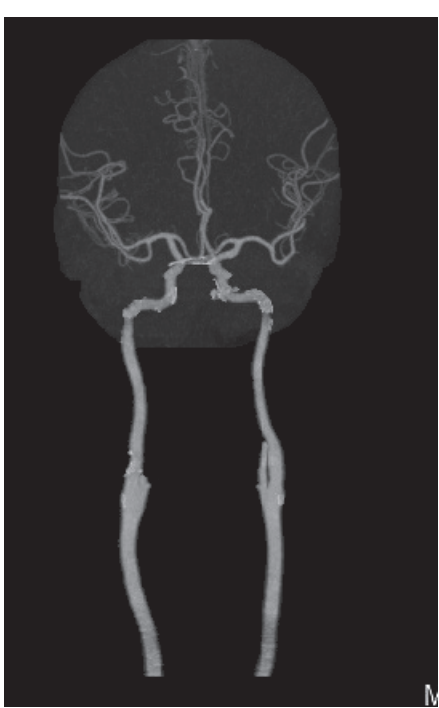

(b)

FIGURE 3: (Day 12) (a) noncontrast CT of the brain demonstrates interval development of acute hemorrhage within medial right temporal lobe, a rare complication of herpes simplex encephalitis. (b) CT angiogram with IV contrast: coronal MIP reconstruction demonstrates patency of the intracranial circulation, with widely patent intracranial arteries, including middle cerebral arteries. Arrows in (a) refer to area of acute hemorrhage within the right medial temporal lobe.

$[7,13]$. Some theories stated that bleeding can be due to rupture and transient hypertension caused by raised intracranial pressure [31]. Another hypothesis includes immune mediated inflammatory reaction that would damage the brain tissue and make it vulnerable to bleeding [13]. The occurrence of the hemorrhage at the site of an abnormal MRI signal suggests that changes in the brain parenchyma and vessels induced by the encephalitis carry a potential risk of spontaneous bleeding [7], so close monitoring of neurological status is recommended in these patients. Fortunately, our patient's neurological status improved to his baseline.

\section{Conclusion}

Intracranial bleeding, although infrequent, is a possible complication of HSV encephalitis even in the setting of early treatment with antiviral. Neurological exam follow-up is essential in these patients. Early imaging studies are required when there is decline in neurological status and/or signs of increased intracranial pressure to rule out intracranial hemorrhage.
Abbreviations
HSV: Herpes simplex virus
CT: Computed tomography
MRI: Magnetic resonance imaging
FLAIR: Fluid attenuated fluid recovery
DWI: Diffusion Weighted Imaging
MIP: Maximum intensity projection
EEG: Electroencephalography
DNA: Deoxyribonucleic acid
WBC: White blood cells

PMN: Polymorph nuclear

ED: Emergency department

CSF: Cerebrospinal fluid

PTT: Partial thromboplastin time

PT: Prothrombin time

INR: International normalized ratio.

\section{Conflicts of Interest}

The authors declare that they have no conflicts of interest.

\section{References}

[1] R. J. Whitley, S. J. Soong, P. R. Dolin, G. J. Galasso, L. T. Ch’ien, and C. A. Alford, "Adenine arabinoside therapy of biopsy proved herpes simplex encephalitis. National institute of allergy and infectious diseases collaborative antiviral study," New England Journal of Medicine, vol. 297, no. 6, pp. 289-294, 1977.

[2] S. B. Snider, C. S. Jacobs, P. S. Scripko, J. P. Klein, and J. L. Lyons, "Hemorrhagic and ischemic stroke secondary to herpes simplex virus type 2 meningitis and vasculopathy," Journal of NeuroVirology, vol. 20, no. 4, pp. 419-422, 2014.

[3] A. Malik, M. Goyal, N. K. Mishra, S. B. Gaikwad, and V. Padma, "Intracerebral haematoma formation in herpes simplex encephalitis: A case report," Australasian Radiology, vol. 41, no. 3, pp. 303-305, 1997.

[4] H. Hiyama, Y. Tanaka, T. Kawakami et al., "A case of fatal herpes encephalitis presenting massive cerebral hematoma," No Shinkei Geka. Neurological Surgery, vol. 29, pp. 271-276, 2001.

[5] E. G. Plantinga and J. A. L. Vanneste, "Mild herpes simplex encephalitis worsening despite acyclovir treatment [6]," Journal of Neurology, vol. 248, no. 3, pp. 237-238, 2001. 
[6] G. Erdem, P. A. Vanderford, and R. D. Bart Jr., "Intracranial hemorrhage in herpes simplex encephalitis: An unusual presentation," Pediatric Neurology, vol. 27, no. 3, pp. 221-223, 2002.

[7] J. M. Politei, I. Demey, and M. A. Pagano, "Cerebral haematoma in the course of herpes simplex encephalitis," Revista de Neurologia, vol. 36, no. 7, pp. 636-639, 2003.

[8] A. Biswas, S. Das, T. Roy, T. Dhibar, and S. Ghorai, "Acute intracerebral hematoma-anunusual presentation of herpes simplex encephalitis," Journal of the Association of Physicians of India, vol. 52, pp. 69-71, 2004.

[9] N. Kabakus, M. K. Gurgoze, H. Yildirim, A. Godekmerdan, and M. Aydin, "Acute hemorrhagic leukoencephalitis manifesting as intracerebral hemorrhage associated with herpes simplex virus type I," Journal of Tropical Pediatrics, vol. 51, no. 4, pp. 245-249, 2005.

[10] R. J. Whitley, "Herpes simplex encephalitis: adolescents and adults," Antiviral Research, vol. 71, no. 2-3, pp. 141-148, 2006.

[11] B. P. Shelley, S. B. Raniga, and J. Al-Khabouri, "An unusual late complication of intracerebral haematoma in herpes encephalitis after successful acyclovir treatment," Journal of the Neurological Sciences, vol. 252, no. 2, pp. 177-180, 2007.

[12] J. Z. Li and P. E. Sax, "HSV-1 encephalitis complicated by cerebral hemorrhage in an HIV-positive person," AIDS Reader, vol. 19, no. 4, pp. 153-155, 2009.

[13] Y. Fukushima, H. Tsuchimochi, M. Hashimoto et al., "A case of herpetic meningoencephalitis associated with massive intracerebral hemorrhage during acyclovir treatment: A rare complication," Neurological Surgery, vol. 38, no. 2, pp. 171-176, 2010.

[14] S. Takeuchi and Y. Takasato, "Herpes simplex virus encephalitis complicated by intracerebral hematoma," Neurology India, vol. 59, no. 4, pp. 594-596, 2011.

[15] W. B. Lo, D. J. Wilcock, M. Carey, and E. Albanese, "Herpes encephalitis complicated by cerebral haemorrhage," Journal of Neurology, Neurosurgery and Psychiatry, vol. 84, no. 12, pp. 1404-1406, 2013.

[16] A. Rodríguez-Sainz, I. Escalza-Cortina, L. Guio-Carrión et al., "Intracerebral hematoma complicating herpes simplex encephalitis," Clinical Neurology and Neurosurgery, vol. 115, no. 10, pp. 2041-2045, 2013.

[17] W. Yu, A. Lee, and B. Welch, "Herpes simplex encephalitis presents as large temporal lobe hemorrhage," Case Reports in Neurology, vol. 1, pp. 12-15, 2014.

[18] S. Zabroug, M. Idalène, S. Azmoun, F. Ihbibane, and N. Tassi, "Postpartum herpetic encephalitis complicated by cerebral hematoma," Revue Neurologique, vol. 171, no. 8-9, pp. 680-682, 2015.

[19] R. R. Mahale, A. Mehta, A. K. Shankar, A. Miryala, P. Acharya, and R. Srinivasa, "Bilateral cerebral hemorrhage in herpes simplex encephalitis: Rare occurrence," Journal of Neurosciences in Rural Practice, vol. 7, no. 5, pp. S128-S130, 2016.

[20] K. Mueller, J. E. Ryan, A. Tai, and R. A. Armonda, "Delayed Temporal Lobe Hemorrhage After Initiation of Acyclovir in an Immunocompetent Patient with Herpes Simplex Virus-2 Encephalitis: A Case Report," Cureus.

[21] R. Whitley, A. D. Lakeman, A. Nahmias, and B. Roizman, "DNA restriction-enzyme analysis of herpes simplex virus isolates obtained from patients with encephalitis," New England Journal of Medicine, vol. 307, no. 17, pp. 1060-1062, 1982.
[22] N. M. Vora, R. C. Holman, J. M. Mehal, C. A. Steiner, J. Blanton, and J. Sejvar, "Burden of encephalitis-associated hospitalizations in the United States, 1998-2010," Neurology, vol. 82, no. 5, pp. 443-451, 2014.

[23] A. J. Nahmias, R. J. Whitley, A. N. Visintine, Y. Takei, and C. A. Alford, "Herpes simplex virus encephalitis: Laboratory evaluations and their diagnostic significance," Journal of Infectious Diseases, vol. 145, no. 6, pp. 829-836, 1982.

[24] R. J. Whitley, S. J. Soong, C. Linneman Jr., C. Liu, G. Pazin, and C. A. Alford, "Herpes simplex encephalitis. Clinical assessment," The Journal of the American Medical Association, vol. 247, no. 3, pp. 317-320, 1982.

[25] A. A. Argyriou, I. Tsota, E. Solomou et al., "Intracerebral haemorrhage as a rare complication of HSV-1 meningoencephalitis: Case report and review of the literature," Scandinavian Journal of Infectious Diseases, vol. 38, no. 1, pp. 63-66, 2006.

[26] A. R. Tunkel, C. A. Glaser, K. C. Bloch et al., “The management of encephalitis: Clinical practice guidelines by the Infectious Diseases Society of America," Clinical Infectious Diseases, vol. 47, no. 3, pp. 303-327, 2008.

[27] P. Hollinger, L. Matter, and M. Sturzenegger, "Normal MRI findings in herpes simplex virus encephalitis [1]," Journal of Neurology, vol. 247, no. 10, pp. 799-801, 2000.

[28] N. D. Marchbank, D. C. Howlett, D. F. Sallomi, and D. V. Hughes, "Magnetic resonance imaging is preferred in diagnosing suspected cerebral infections," British Medical Journal, vol. 320, no. 7228, pp. 187-188, 2000.

[29] R. N. Sener, "Herpes simplex encephalitis: Diffusion MR imaging findings," Computerized Medical Imaging and Graphics, vol. 25, no. 5, pp. 391-397, 2001.

[30] C.-W. Lai and M. E. Gragasin, "Electroencephalography in Herpes Simplex Encephalitis," Journal of Clinical Neurophysiology, vol. 5, no. 1, pp. 87-104, 1988.

[31] H.-J. Yan, "Herpes simplex encephalitis: The role of surgical decompression," Surgical Neurology, vol. 57, no. 1, pp. 20-24, 2002. 


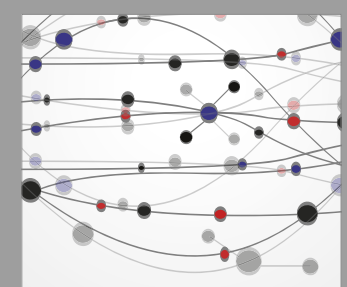

The Scientific World Journal
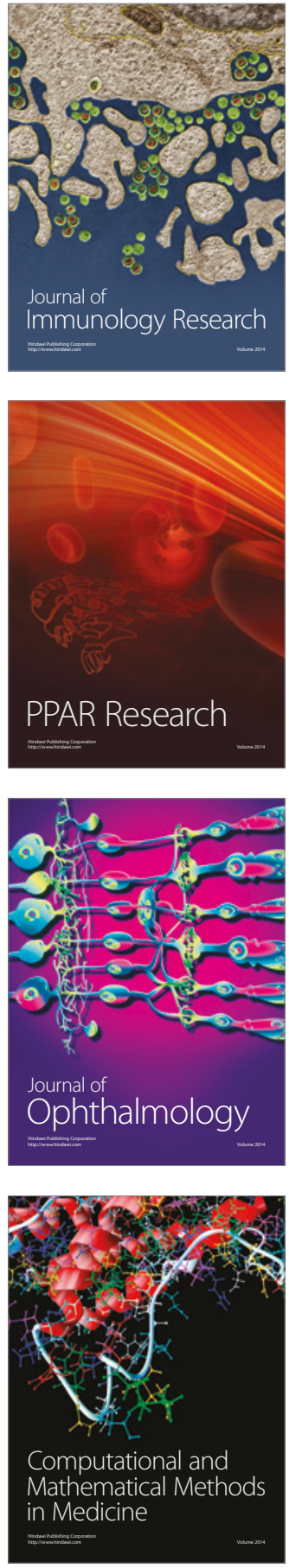

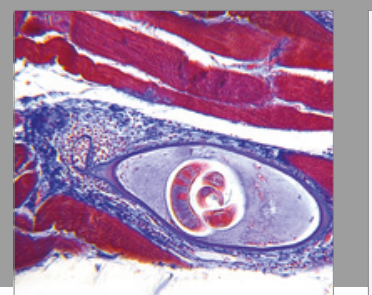

Gastroenterology Research and Practice
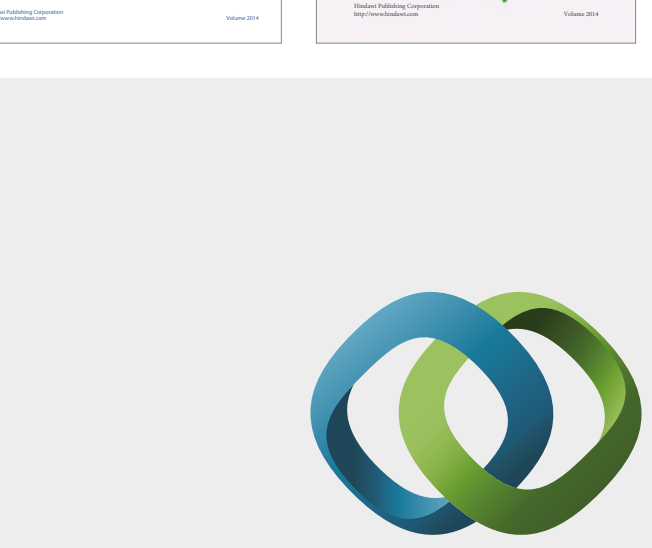

\section{Hindawi}

Submit your manuscripts at

https://www.hindawi.com
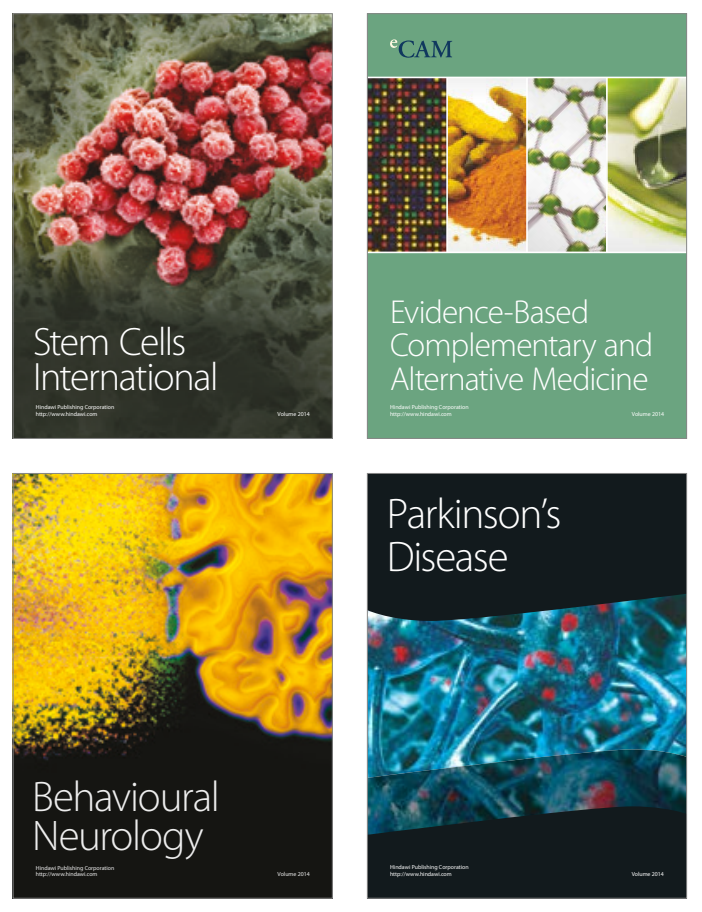
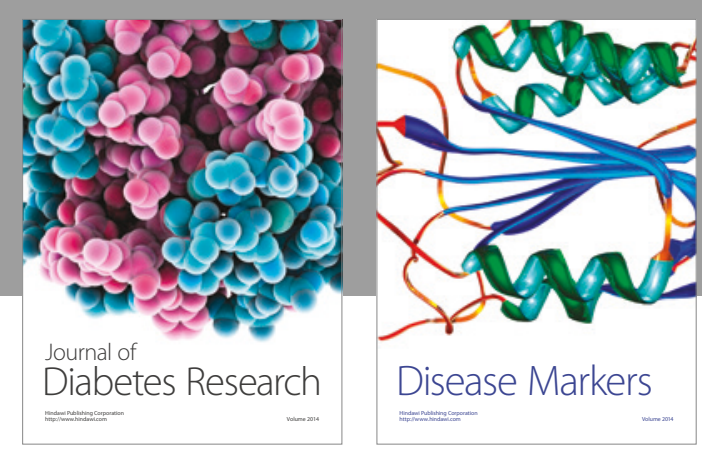

Disease Markers
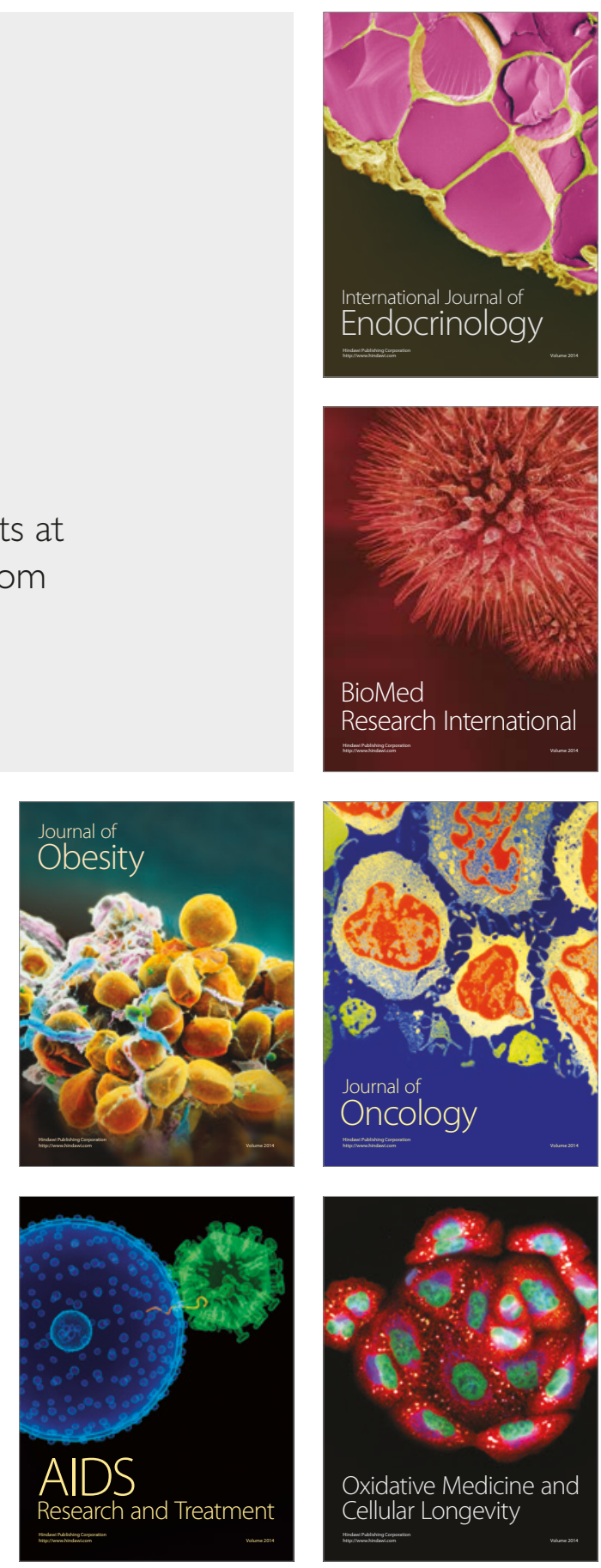